\title{
Plasma-phototransducer of Solar Energy into Electricity
}

\author{
A. Aramyan ${ }^{1, *}$, S. $\operatorname{Aramyan}^{2}$, Y. Martinez S. ${ }^{3}$ \\ ${ }^{1}$ Institute of Applied Problems of Physics of National Academy of Sciences of Armenia, Hr. Nersessian Street 25, 0014 Yerevan, Republic \\ of Armenia \\ ${ }^{2}$ International Scientific and Educational Center of National Academy of Sciences of Armenia, Marshal Baghramian Ave. $24 \mathrm{~d}, 0019$ \\ Yerevan, Republic of Armenia \\ ${ }^{3}$ Instituto Tecnológico y de Estudios Superiores de Occidente, ITESO, Tlaquepaque, Jalisco, México \\ *Corresponding Author: aramyan.artur@gmail.com
}

Copyright (C) 2013 Horizon Research Publishing All rights reserved.

\begin{abstract}
The aim of this article is to describe a new direct converter form solar energy to electricity. This system has the convenience that there are not released toxic gases or any other greenhouse effects. Additionally, it is constructed with common materials such as $\mathrm{NaCl}$, or other salty components, and it is relatively easy to manufacture.
\end{abstract}

Keywords Low-Temperature Plasma, Solar Energy Converter, Photoionisation, Alkali Metals, Ambipolar Potential

\section{Introduction}

Using a low-temperature plasma for the direct conversion of light energy into electrical energy studied very little. It is known a proposal for a converter of solar energy into electricity based on magnetohydrodynamic (MHD) conversion in an isothermal plasma alkali metals [1]. Another method for the direct conversion of the kinetic energy of charged particles in to the electricity in the plasma is the use of the ambipolar potential difference. The formation of ambipolar potential difference in the photoplasm is similar to the formation of photo-emf by inhomogeneous excitation of a homogeneous semiconductor. Partial distribution of charges is due to the difference in the mobility of positive ions (holes in semiconductors) and electrons. More mobile electrons quickly leave the area of the primary ionization. Due to this, there is the ambipolar potential difference, the value of which determines the value of the photo-emf. The hole mobility is different from the electron mobility. However, this difference does not exceed an order of magnitude due to the influence of the intracrystalline field in the semiconductor [2].

\section{Theoretical Analysis and Estimations}

In plasma, the difference in the values of mobility of the electrons and the ions is determined by the ratio of the mass of the charged particles $\sqrt{M / m} \sim 100$, which is an order of magnitude higher than the similar value in semiconductors. This property of a low-temperature plasma defines more effective charge separation compared with semiconductors. Experimental studies on the use of the ambipolar potential difference in photoplasm of alkali metals for the direct conversion of the energy of the concentrated radiation into electricity started not long ago. The process of formation of the plasma in the vapors of alkali metals is a well-studied phenomenon. The efficiency of this process increases by several orders of magnitude when the wavelength of the incident radiation corresponds to the absorption lines of alkali atoms. Even in the first papers on photoplasm was shown that the conversion factor of the radiation energy into the ionization energy can be close to unity. In work [3] shows that the resonantly excited sodium atoms quite long-lived. The excitation was performed using a laser beam in which the wavelength of $5900 \AA$. This corresponds to the center of the solar radiation spectrum. At the same time, in works $[4,5]$ shows that the highly excited atoms are long-lived too. As known from the quantum theory The average lifetime of $t_{n}$ depends on the value of the first quantum number $\mathrm{n}$ as $t_{n} \sim n^{4}$.

An interesting feature of the measurements was that the magnitude of the photovoltage in plasma was $3-4 \mathrm{~V}$, which is almost an order of magnitude higher compared to the semiconductor elements. The above values of photo-emf are the result of electrons' high temperature, which have been measured in photoplasm.

The resulting ambipolar electric field determines the photovoltage. Ambipolar field intensity determined by the known expression:

$$
E_{a} \approx-T_{e} \frac{\operatorname{grad}(n)}{n}
$$

where $T_{e}$ - the electron temperature, $n$ - density of charged particles.

From (1) is seen that the effective charge separation is initially started by the growth of the electron temperature 
and the high value of charged gradient of particles. Temperature of electrons in semiconductor converters is usually close to the temperature of the crystal. It is known that the efficiency of a silicon transmitter falls to zero at $300^{\circ} \mathrm{C}$. Therefore, an important role in shaping the voltage in the semiconductor converters is the high value of the density gradient of the charged particles, which is achieved through the creation of artificial inhomogeneities: p-n-transition, heterostructures, etc.

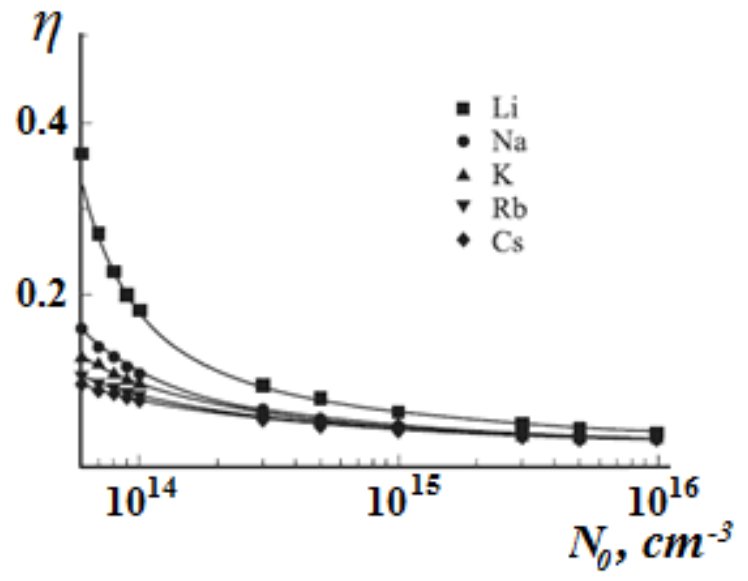

Figure 1. The efficiency of conversion for different alkali metals, depending on the density of the vapor of alkali metals.

In photoplasma is possible the realization of much higher temperatures. Any substance under intense heat tends to move to the plasma state. Electron temperature in plasma tends to the thermodynamic limit $T_{t d}$, determined by the temperature of optical radiation source. The emission spectrum of the sun is close to the emission spectrum of an absolutely black body, heated to a temperature of $5800^{\circ} \mathrm{K}$, which determines the high value of the thermodynamic limit of the conversion efficiency of solar radiation $[6,7]$.

In demonstration experiments in search of the photovoltaic effect in plasma was recorded value of photovoltage about $4 \mathrm{~V}$, which an order of magnitude greater than silicon solar cells. Accordingly, the value of such a large photovoltage is due to the high electron temperature $\left(4000-10000^{\circ} \mathrm{K}\right)[8]$.

In calculation of $T_{e}$ has been assumed that the density of alkali atoms $N_{o}$ corresponds to the vapor pressure. As can be seen, with the same external conditions, the greatest efficiency is achieved in the pairs of lithium since for lithium are the maximum values of $T_{e}$. The evaluation of maximum possible efficiency of phototransducer, with planar electrodes is given by

$$
\eta=\frac{1}{4} \frac{T_{e}}{\Delta E_{01}} \ln 3
$$

where $\Delta E_{01}$ - the difference between the energy of the first resonance levels in alkali atoms. From this formula it is clear that to improve the conversion efficiency in photoplasma, is important to achieve high-temperature electron gas.

As is known, the electron temperature $T_{e}$ depends on the density of neutral atoms $N_{0}$, according to this expression

$$
T_{e}=\frac{e}{\sigma_{t r} \sqrt{\delta}} \frac{E}{N_{0}}
$$

where $e$ - electron charge, $E$ - electrical field, $\sigma_{t r}$ - scattering cross section, $\delta=2 \mathrm{~m} / \mathrm{M}$ (m- electron mass and M- atom mass).

Using (2) and (3) one can present conversion efficiency for the various alkali metal, depending on the density of the alkali metal vapor.

The emergence of the photovoltage in the plasma is the result of the ambipolar field in the asymmetric arrangement of the electrodes relative to the excitation by an external source of light. The electric current flowing through the external circuit is determined by the diffusion of positive ions, i.e., the same as in semiconductors, the diffusion of non-major carriers. The growth of the electron temperature increases photovoltage values and conversion efficiency.

To increase the conversion efficiency can also be used mixtures of different alkali metals, which will enable the use of sunlight more efficiently. The use of acoustic waves will also help the conversion. Phase shift between the electron and ion fluctuations leads to the separation of charges, which will increase the ambipolar field. The increase in the field will increase the photovoltage [9-12].

And instead of pure alkali metals can be used salts of these metals. According to [13] can be argued that in the vapor of alkali metal salts, is enough pure alkali atoms. To confirm that the alkali metals can be easily obtained, can perform a simple test, just throw a simple salt $(\mathrm{NaCl})$ in any flame (for example alcohol lamp where the temperature is $600-700^{\circ} \mathrm{C}$ ) and will seen the yellow colour flame which corresponds to sodium spectrum. It turns out that at such a low temperature can be obtained even of excited atoms of alkali metals from their salts. On the other side, this will lead to the expansion of range of sunlight absorption. To extend the optical absorption bands can be used molecular additives, which absorption range corresponds to the maximum radiation of the sun.

\section{Conclusions}

Thus, we can draw the following conclusion. Using the effect of photoionization and fact of nonequilibrium of energy between electrons and ions can be argued that possible to get EMF in photoplasm of vapors of alkali metals. To increase efficiency of converter encouraged to use salts of alkali metals.

\section{REFERENCES}


[1] Terry J. Hendricks, Chendong Huang. High-Performance radial AMTEC cell desing for ultra-high-power solar AMTEC systems, J. of Solar Energy Engineering. Vol.122. No.2. 49-55.

[2] G. J. Dunning, A. J. Palmer, Towards a high-temperature solar electric converter, J. Appl.Phys. Vol.52. No.12. 7086-7091.

[3] V.M. Arutunyan, A.R. Aramyan, et. al. Polarimetric investigation of relaxation times of $3 \mathrm{P} 1 / 2,3 / 2$ excited levels in dense vapors of atomic sodium. Sov. J. of Contemporary Phys., Vol.21, No.2, 58-62.

[4] A.R. Aramyan, Acoustic Impact on Superluminescence in Argon Plasma, JETP Letters, Vol.77, No.6, 326-329.

[5] A.R. Aramyan, Observations of Acoustic-Wave-Induced Superluminescence in an Argon Plasma., Physical Review Letters, Vol.91, No15, 155002-1-155002-4.

[6] N. A. Leontovich, Maximum efficiency of direct utilization of radiation. Sov. Phys. Usp. Vol.18, 963-964.

[7] I. I. Sobel'man, Exploitation of solar energy. Sov. Phys. Usp.
Vol.19, 758-764.

[8] N. A. Gorbunov and T. Stacewicz. Photo EMF observed upon the laser resonance excitation of sodium vapors. Technical Physics Letters, Vol.26, No.8, 654-655.

[9] A.R. Aramyan, G.A. Galechyan, A.R. Mkrtchyan, Plasma in the field of an intesne sound wave, Soviet physics. Acoustics (Sov.phys.Acoust.), vol.37, no2, 107-118.

[10] A. Aramyan, G. Galechyan, Characteristics of sound wave generation in a gas discharge. Tech. Phys. Vol.42, No.8, 901-904.

[11] A.R. Aramyan, G.A. Galechyan, A.R. Mkrtchyan, SU patent № 1664104 A1 (1991).

[12] A.R. Aramyan, G.A. Galechyan, Vortices in gas-discharge plasma, Physics-Uspekhi Vol.50, No.11, 1147-1171.

[13] V.M. Arutunyan, I.G. Arutunyan, T.A. Papazyan, S.P. Ishkhanyan. Determination of the density of neutral sodium atoms in laser plasmas by polarization method. Bulletin of the USSR Academy of Sciences. Physic, Vol.47, No.12, 2420-2426. 